\title{
Similarities and differences between positive and negative particle masses in the bicubic equation limiting particle velocity formalism: positive or negative muon neutrino mass?
}

\author{
Josip Śoln ${ }^{1}$
}

\author{
${ }^{1}$ JZS Phys-Tech, Vienna, Virginia 22182, USA \\ Correspondence: Josip Śoln, JZS Phys-Tech, Vienna, Virginia 22182, USA. E-mail: jurasoln@ yahoo.com
}

Received: August 27, 2018

Accepted: September 12, 2018

Online Published: September 27, 2018

doi:10.5539/apr.v10n5p40

URL: https://doi.org/10.5539/apr.v10n5p40

\begin{abstract}
Here, rather detailed numerical comparisons of energies and momenta for positive $m_{+}=m>0$ and negative $m_{-}=-m<$ 0 particle masses with $m_{+}^{2}=m_{-}^{2}=m^{2}$, within the bicubic equation limiting particle velocity formalism with three particle limiting velocities $c_{1}, c_{2}$ and $c_{3}$, are done. Already these limiting velocities, on a global scale, can differentiate positive, $m_{+}$and negative $m_{-}$particle masses. While $c_{1}\left(m_{+}\right), c_{2}\left(m_{+}\right)$and $c_{3}\left(m_{+}\right)$are real, imaginary and real, corresponding, respectively, to primary, obscure and normal particles; $c_{1}\left(m_{-}\right), c_{2}\left(m_{-}\right)$and $c_{3}\left(m_{-}\right)$are respectively imaginary, real and real, now representing respectively, obscure, primary and normal particles. In fact, from limiting velocity solutions, one identifies: $c_{1}^{2}\left(m_{+}\right)=c_{2}^{2}\left(m_{-}\right), c_{1}^{2}\left(m_{-}\right)=c_{2}^{2}\left(m_{+}\right), c_{3}^{2}\left(m_{-}\right)=c_{3}^{2}\left(m_{+}\right)$. The unified particle mass-shell like forms with particle energies and momenta are readily expressible for $m_{+}=m>0$ and $m_{-}=-m<0$ masses with respective limiting velocities, separating $c_{3}\left(m_{+}\right)$from $c_{3}\left(m_{-}\right)$as well as $c_{1}\left(m_{+}\right)$from $c_{1}\left(m_{-}\right)$and $c_{2}\left(m_{+}\right)$from $c_{2}\left(m_{-}\right)$.

We assume that flavor neutrinos, which, while in process do not change flavor, belong to normal limiting velocity $c_{3}$ class. Then the muon neutrino from OPERA velocity measurement should maintain the same velocity squares $v^{2}$ and $c_{3}^{2}$ when one changes the positive neutrino mass $m_{+v}(\mu)>0$ into the negative neutrino mass $m_{-v}(\mu)<0$, since theoretically $c_{3}^{2}\left(m_{+v}(\mu)\right)=c_{3}^{2}\left(m_{-v}(\mu)\right)$. For OPERA measurements this is verified perturbatively by simultaneously evaluating squares of normal limiting velocities with $m_{+v}(\mu)$ and $m_{-v}(\mu)$ masses, yielding the same result $c_{3}^{2}\left(m_{+v}(\mu)=c_{3}^{2}\left(m_{-v}(\mu) \simeq v_{v}^{2}(\mu) \simeq\right.\right.$ $c^{2}$.
\end{abstract}

Keywords: particle masses, muon neutrino mass, bicubic equation limiting particle velocity formalism

\section{Introduction}

The bicubic equation limiting particle velocity formalism ('Soln, 2014, 2015, 2016, 2017) evolved to the point that it was straightforward to indicate the possibility of a negative particle mass ('Soln, 2018) as discussed by Gaal (2017) and experimentally observed by Khamehchi et al. (2017) in their "Negative mass hydrodynamics in a spin-orbit-coupled Bose-Einstein condensate".

The congruent parameter $z=3 \sqrt{3} m v^{2} / 2 E$ (with $m, v$, and $E$ respectively, as particle mass, velocity and energy) in the bicubic equation limiting particle velocity formalism can assume values as $-1 \leq z(m) \leq 1$. The positive branch $0 \leq z \leq 1$, practically demands $m \geq 0, v^{2} \geq 0$ and $E \geq 0$. The negative branch $-1 \leq z \leq 0$, if necessary, is easily satisfied with $m \leq 0$ but keeping $v^{2} \geq 0$ and $E \geq 0$. Hence, with $v^{2} \geq 0$ and $E \geq 0$ and with two kinds of masses $m_{+}=m \geq 0$, and $m_{-}=-m \leq 0$, one covers the whole allowed values of the congruent parameter $-1 \leq z(m) \leq 1$.

With these preliminaries, the biqubic equation limiting velocity formalism is used to explicitly evaluate energies and momenta for particles with three limiting velocities $c_{1}, c_{2}$ and $c_{3}$, separately for positive and negative particle masses $m_{+}=m \geq 0, m_{-}=-m \leq 0$. This results jn detailed energy and momentum expressions for every $m_{+}$and $m_{-}$massive particle for each corresponding limiting velocity $c_{i}\left(m_{+}\right)$and $c_{i}\left(m_{-}\right)$with $i=1,2,3$, whose comparisons show similarities and differences among them. Noticeable being $c_{1}^{2}\left(m_{+}\right)=c_{2}^{2}\left(m_{-}\right), c_{1}^{2}\left(m_{-}\right)=c_{2}^{2}\left(m_{+}\right)$and $c_{3}^{2}\left(m_{+}\right)=c_{3}^{2}\left(m_{-}\right)$, with $m_{+,-}= \pm m$, $m \geq 0$.

Finally, one can address the question as to the limiting velocity of the muon neutrino $v(\mu)$ from the OPERA measurement (Adam et al., 2013) and analyses in 'Soln $(2014,2016)$ here denoted as $c_{3}\left(m_{\nu}(\mu)\right.$. Then with presumed positive and negative neutrino masses $m_{+v}(\mu) \geq 0$ and $m_{-v}(\mu) \leq 0$ the values of $c_{3}^{2}\left(m_{+v}(\mu)\right)$ and $c_{3}^{2}\left(m_{-v}(\mu)\right)$ should be the same. Perturbativelly, that turns out to be the case with the result $c_{3}^{2}\left(m_{+v}(\mu)\right)=c_{3}^{2}\left(m_{-v}(\mu)\right) \simeq v_{v}^{2}(\mu) \simeq c^{2}$, where $v_{v}(\mu)$ and $c$ are, respectively the muon neutrino velocity and the velocity of light. 
In Section 2 the bicubic equation limiting particle velocity formalism is briefly exposed from which in table forms the limiting velocity squares $c_{1}^{2}, c_{2}^{2}$ and $c_{3}^{2}$ are presented for $m_{+}=m>0$ and $m_{-}=-m<0$ respectively as functions of congruent parameters satisfying simplified restrictive inequalities $10^{-6} \leq z\left(m_{+}\right) \leq 1$ and $-1 \leq z\left(m_{-}\right) \leq-10^{-6}$, although theoretically, $0 \leq z\left(m_{+}\right) \leq 1$ and $-1 \leq z\left(m_{-}\right) \leq 0$, and for any unrestricted $m$, one has generally $-1 \leq z(m) \leq 1$.

Section 3 is devoted to analyzing energy-momentum relations for $m_{+}=m \geq 0$ and $m_{-}=-m \leq 0$ mass particles. Each of them with three limiting velocities $c_{i}\left(m_{+v}(\mu)\right)$ and $c_{i}\left(m_{-v}(\mu)\right), i=1,2,3$, together with corresponding energies and momenta; this directly leads to calculating the squares of limiting velocity $c_{3}$, for both positive and negative masses $c_{3}^{2}\left(m_{+v}(\mu)\right)$ and $c_{3}^{2}\left(m_{-v}(\mu)\right)$, whose equality has to be respected when $c_{3}$ is involved in the bicubic equation limiting particle velocity formalism.

Conclusion with final remarks is given in Section 4.

\section{Energies and momenta expressions with positive and negative particle masses via the corresponding congruent parameters}

As shown in references ('Soln, 2014, 2015, 2016, 2017, 2018), for the sake of completeness, the best thing is to start with the bicubic equation for the particle limiting velocity,generically denoted with $c$,

$$
m^{2}\left(c^{2}\right)^{3}-E^{2} c^{2}+E^{2} v^{2}=0
$$

where a particle mass squared $m^{2}$ represents both positive and negative mass, $m_{+}=m \geq 0$ and $m_{-}=-m \leq 0$ with properties $m_{ \pm}^{2}=m^{2}$ as shown in 'Soln (2018). As usual, $E$ and $v$ are particle energy and velocity, respectively. The three solutions, denoted as $c_{i}, i=1,2,3$, expressed in the squares forms, reflect separate dependences on $m_{+}$and $m_{-}$ particle masses. Next with the help of congruent parameters, we show that solutions that follow are characterized by the discriminant satisfying $D<0$

$$
\begin{aligned}
m_{ \pm} & = \pm m, m \geq 0, z\left(m_{ \pm}\right)=\frac{3 \sqrt{3} m_{ \pm} v^{2}}{2 E}, \\
-1 & \leq z\left(m_{ \pm}\right) \leq 1,0 \leq z\left(m_{+}\right) \leq 1,-1 \leq z\left(m_{-}\right) \leq 0, \\
D\left(m_{ \pm}\right) & =\left(\frac{27}{8}\right)^{2} \frac{1}{z\left(m_{ \pm}\right)^{4}}\left(1-\frac{1}{z\left(m_{ \pm}\right)^{2}}\right) \prec 0
\end{aligned}
$$

The discriminant, $D \prec 0$, allows us now to write down the solutions separately with $m_{+}$and $m_{-}$, expressed with the help of $m$.

Now, according to 'Soln (2018) the solutions of (1) with notations from (2), in the square forms, are

$$
\begin{aligned}
\frac{c_{1}^{2}\left(m_{+}\right)}{v^{2}} & =\frac{3}{z\left(m_{+}\right)} \sin \left[\frac{1}{3}\left(\pi-\sin ^{-1}\left(z\left(m_{+}\right)\right)\right)\right] \\
& =\frac{3}{z(m)} \sin \left[\frac{1}{3}\left(\pi-\sin ^{-1}(z(m))\right)\right]>0 ; \\
\frac{c_{2}^{2}\left(m_{+}\right)}{v^{2}} & =-\frac{3}{z\left(m_{+}\right)} \sin \left[\frac{1}{3}\left(\pi+\sin ^{-1}\left(z\left(m_{+}\right)\right)\right)\right] \\
& =-\frac{3}{z(m)} \sin \left[\frac{1}{3}\left(\pi+\sin ^{-1}(z(m))\right)\right]<0 ; \\
\frac{c_{3}^{2}\left(m_{+}\right)}{v^{2}} & =\frac{3}{z\left(m_{+}\right)} \sin \left[\frac{1}{3} \sin ^{-1}\left(z\left(m_{+}\right)\right)\right] \\
& =\frac{3}{z(m)} \sin \left[\frac{1}{3} \sin ^{-1}(z(m))\right]>0 .
\end{aligned}
$$




$$
\begin{aligned}
\frac{c_{1}^{2}\left(m_{-}\right)}{v^{2}} & =\frac{3}{z\left(m_{-}\right)} \sin \left[\frac{1}{3}\left(\pi-\sin ^{-1}\left(z\left(m_{-}\right)\right)\right)\right] \\
& =-\frac{3}{z(m)} \sin \left[\frac{1}{3}\left(\pi+\sin ^{-1}(z(m))\right)\right]<0, \\
\frac{c_{2}^{2}\left(m_{-}\right)}{v^{2}} & =-\frac{3}{z\left(m_{-}\right)} \sin \left[\frac{1}{3}\left(\pi+\sin ^{-1}\left(z\left(m_{-}\right)\right)\right)\right] \\
& =\frac{3}{z(m)} \sin \left[\frac{1}{3}\left(\pi-\sin ^{-1}(z(m))\right)\right]>0, \\
\frac{c_{3}^{2}\left(m_{-}\right)}{v^{2}} & =\frac{3}{z\left(m_{-}\right)} \sin \left[\frac{1}{3} \sin ^{-1}\left(z\left(m_{-}\right)\right)\right] \\
& =\frac{3}{z(m)} \sin \left[\frac{1}{3} \sin ^{-1}(z(m))\right]>0 .
\end{aligned}
$$

While the $c_{1}\left(m_{+}\right), c_{2}\left(m_{+}\right)$and,$c_{3}\left(m_{+}\right)$are limiting velocities, respectively of particles named primary, obscure and normal particle, the $c_{1}\left(m_{-}\right), c_{2}\left(m_{-}\right)$and $c_{3}(m-)$ are limiting velocities, respectively of particles named now obscure, primary and normal particle. As every limited velocity solution $c_{i}, i=1,2,3$ in (3) and (4) has been expressed in terms of particle common mass $m$, then directly from solutions (3) and (4) one deduces identities among the squares of limiting velocities,

$$
\begin{aligned}
& c_{1}^{2}\left(m_{+}\right)=c_{2}^{2}\left(m_{-}\right)>0, \\
& c_{2}^{2}\left(m_{+}\right)=c_{1}^{2}\left(m_{-}\right)<0, \\
& c_{3}^{2}\left(m_{+}\right)=c_{3}^{2}\left(m_{-}\right)>0
\end{aligned}
$$

The expressions (5) of equal values of squares of limiting velocities with $m_{+}$and $m_{-}$masses imply also the equal values of $E, m^{2}$ and $v^{2}$ with which one might regenerate each other. Formally, for a particle with either $m_{+}$or $m_{-}$mass one has the relations connecting energies with masses and velocity

$$
E\left(m_{ \pm}\right)=\frac{3 \sqrt{3} m_{ \pm} v^{2}}{2 z\left(m_{ \pm}\right)}>0
$$

The idea here is to express, respectively $v^{2} / z\left(m_{+}\right)$and $v^{2} / z\left(m_{-}\right)$in terms of squares of limiting velocity solutions $c_{i}^{2}\left(m_{+}\right)$ and $c_{i}^{2}\left(m_{-}\right), i=1,2,3$. These, according to the 'Soln (2016), utilizing the $z-$ algebra, will yield not only energies $E\left(c_{i}\left(m_{ \pm}\right)\right)$but also the momenta $\vec{p}\left(c_{i}\left(m_{ \pm}\right)\right), i=12,3$.

An example of utilizing relation (6) to obtain specifically $E\left(c_{1}\left(m_{-}\right)\right)$and $\vec{p}\left(c_{1}\left(m_{-}\right)\right)$with the help of the $z-$ algebra from 'Soln (2016) is exhibited next,

$$
\begin{gathered}
E\left(c_{1}\left(m_{-}\right)\right)=\frac{\sqrt{3}\left(m_{-}\right) c_{1}^{2}\left(m_{-}\right)}{2 \sin \left(\frac{1}{3}\left(\pi-\sin ^{-1}\left(z\left(m_{-}\right)\right)\right)\right.} \\
=m_{-} c_{1}^{2}\left(m_{-}\right)\left(1-\frac{v^{2}}{c_{1}^{2}\left(m_{-}\right)}\right)^{-\frac{1}{2}}, \\
\vec{p}\left(c_{1}\left(m_{-}\right)\right)=\frac{E\left(c_{1}\left(m_{-}\right)\right.}{\left(-c_{1}^{2}\left(m_{-}\right)\right)}=\left(-m_{-}\right) \vec{v}\left(1-\frac{v^{2}}{c_{1}^{2}\left(m_{-}\right)}\right)^{-\frac{1}{2}}
\end{gathered}
$$

In 'Soln (2016) $E\left(c_{i}\left(m_{+}\right)\right.$and $\vec{p}\left(c_{i}\left(m_{+}\right), i=1,2,3\right.$, have been already evaluated, while in 'Soln (2018) only $E\left(c_{i}\left(m_{-}\right)\right.$, $i=1,2,3$. Here, one completes the job with negative masses $m_{-}=-m<0$ by adding also $\vec{p}\left(c_{i}\left(m_{-}\right), i=1,2,3\right.$, so that 
for both $m_{+}>0$ and $m_{-}<0$ masses unified particle mass shell like forms with respective limiting velocities $c_{i}\left(m_{+}\right)$and $c_{i}\left(m_{-}\right), i=1,2,3$, are exhibited.

The relations (5) indicate that the interrelationship exist between quantities involving masses $m_{+}=m>0$ and $m_{-}=-m<$ 0 . To this end, to have notation similar, we rewrite energies and momenta from 'Soln (2016) for $m_{+}=m>0$ masses in the notation from 'Soln (2018). Here, the limiting velocity solutions $c_{i}\left(m_{+}\right), i=12,3$ from 'Soln (2016) with the help of he $z$-algebra from 'Soln $(2016,2018)$ yield the equal value (6) energies with the corresponding momenta for $m_{+}=m>0$ masses in the updated notations:

$$
\begin{aligned}
E\left(c_{1}\left(m_{+}\right)\right) & =m_{+} c_{i}^{2}\left(m_{+}\right)\left(1-\frac{\vec{v}^{2}}{c_{1}^{2}\left(m_{+}\right)}\right)^{-\frac{1}{2}}, \\
\vec{p}\left(c_{1}\left(m_{+}\right)\right) & =\frac{E\left(c_{1}\left(m_{+}\right)\right) \vec{v}}{c_{1}^{2}\left(m_{+}\right)}=m_{+} \vec{v}\left(1-\frac{\vec{v}^{2}}{c_{1}^{2}\left(m_{+}\right)}\right)^{-\frac{1}{2}} ; \\
E\left(c_{2}\left(m_{+}\right)\right) & =-m_{+} c_{2}^{2}\left(m_{+}\right)\left(1-\frac{\vec{v}^{2}}{c_{2}^{2}\left(m_{+}\right)}\right)^{-\frac{1}{2}}, \\
\vec{p}\left(c_{2}\left(m_{+}\right)\right. & =\frac{E\left(c_{2}\left(m_{+}\right)\right) \vec{v}}{\left(-c_{2}^{2}\left(m_{+}\right)\right)}=m_{+} \vec{v}\left(1-\frac{\vec{v}^{2}}{c_{2}^{2}\left(m_{+}\right)}\right)^{-\frac{1}{2}} ; \\
E\left(c_{3}\left(m_{+}\right)\right) & =m_{+} c_{3}^{2}\left(m_{+}\right)\left(1-\frac{\vec{v}^{2}}{c_{3}^{2}\left(m_{+}\right)}\right)^{-\frac{1}{2}}, \\
\vec{p}\left(c_{3}\left(m_{+}\right)\right) & =\frac{E\left(c_{3}\left(m_{+}\right)\right) \vec{v}}{c_{3}^{2}\left(m_{+}\right)}=m_{+} \vec{v}\left(1-\frac{\vec{v}^{2}}{c_{3}^{2}\left(m_{+}\right)}\right)^{-\frac{1}{2}} . \\
\vec{p}^{2}\left(c_{i}\left(m_{+}\right)\right) c_{i}^{2}\left(m_{+}\right)-E^{2}\left(c_{i}\left(m_{+}\right)\right)+m_{+}^{2} c_{i}^{4}\left(m_{+}\right) \quad=0, i=1,2,3 . & 0, c_{2}^{2}\left(m_{+}\right)<0, c_{3}^{2}\left(m_{+}\right)>0,
\end{aligned}
$$

The relation (7.2) is a particle mass shell like form for all particle kinds, primary, obscure and normal with respective limiting velocities $c_{i}\left(m_{+}\right), i=1,2,3$ as exhibited in (7.1).

Now,from the relations in (5) of equalities between limiting velocities with $m_{+}=m>0$ and $m_{-}=-m<0$ masses, relation (6) suggests that $E\left(c_{2}\left(m_{-}\right)\right)$and $E\left(c_{1}\left(m_{+}\right)\right)$as well as $\vec{p}\left(c_{2}\left(m_{-}\right)\right.$an $\mathrm{d} \vec{p}\left(c_{1}\left(m_{+}\right)\right.$should be related by the interchange transforms, with having either explicit or implicit exchanges between $m_{+}$and $m_{-}$,

$$
\begin{aligned}
& \text { Explicit } \quad: \quad m_{+} \longleftrightarrow-m_{-} \\
& \text {Implicit : } \\
& c_{1}\left(m_{+}\right) \longleftrightarrow c_{2}\left(m_{-}\right), \\
& c_{2}\left(m_{+}\right) \longleftrightarrow c_{1}\left(m_{-}\right), \\
& c_{3}\left(m_{+}\right) \longleftrightarrow c_{3}\left(m_{-}\right)
\end{aligned}
$$

Applying relations (8) systematically to relations (7.1) and (7.2) one arrives at equal value energies with the corresponding momenta for $m_{-}=-m<0$, in updated notation: 


$$
\begin{aligned}
& E\left(c_{2}\left(m_{-}\right)\right)=-m_{-} c_{2}^{2}\left(m_{-}\right)\left(1-\frac{\vec{v}^{2}}{c_{2}^{2}\left(m_{-}\right)}\right)^{-\frac{1}{2}}, \\
& \vec{p}\left(c_{2}\left(m_{-}\right)\right.=\frac{E\left(c_{2}\left(m_{-}\right)\right) \vec{v}}{c_{2}^{2}\left(m_{-}\right)}=-m_{-} \vec{v}\left(1-\frac{\vec{v}^{2}}{c_{2}^{2}\left(m_{-}\right)}\right)^{-\frac{1}{2}} ; \\
& E\left(c_{1}\left(m_{-}\right)\right)=m_{-} c_{i}^{2}\left(m_{-}\right)\left(1-\frac{\vec{v}^{2}}{c_{1}^{2}\left(m_{-}\right)}\right)^{-\frac{1}{2}}, \\
& \vec{p}\left(c_{1}\left(m_{-}\right)\right)=\frac{E\left(c_{1}\left(m_{-}\right)\right) \vec{v}}{\left(-c_{1}^{2}\left(m_{-}\right)\right)}=-m_{-} \vec{v}\left(1-\frac{\vec{v}^{2}}{c_{1}^{2}\left(m_{-}\right)}\right)^{-\frac{1}{2}} ; \\
& E\left(c_{3}\left(m_{-}\right)\right)=-m_{-} c_{3}^{2}\left(m_{-}\right)\left(1-\frac{\vec{v}^{2}}{c_{3}^{2}\left(m_{-}\right)}\right)^{-\frac{1}{2}}, \\
& \vec{p}\left(c_{3}\left(m_{-}\right)\right)=\frac{E\left(c_{3}\left(m_{-}\right)\right) \vec{v}}{c_{3}^{2}\left(m_{-}\right)}=-m_{-} \vec{v}\left(1-\frac{\vec{v}^{2}}{c_{3}^{2}\left(m_{-}\right)}\right)^{-\frac{1}{2}} . \\
& \vec{p}^{2}\left(c_{i}\left(m_{-}\right)\right) c_{i}^{2}\left(m_{-}\right)-E^{2}\left(c_{i}\left(m_{-}\right)\right)+m_{-}^{2} c_{i}^{4}\left(m_{-}\right) \quad=0, i=1,2,3 .
\end{aligned}
$$

The last relation in (9.2) is unified particle mass-shell like form for all particle kinds, now, $i=1$, obscure, $i=2$, primary and, $i=3$, normal, with respective limiting velocities, $c_{1}\left(m_{-}\right), c_{2}\left(m_{-}\right)$and $c_{3}\left(m_{-}\right)$.

2.1 Numerical procedures with positive and negative mass particle limiting velocities utilizing the congruent parameter $z$

One starts with selected exemplary values for $c_{i}^{2}\left(m_{+}\right) / v^{2}$ and $c_{i}^{2}\left(m_{-}\right) / v^{2}, i=1,2,3$ derived respectively from representative values of $0<z\left(m_{+}\right) \leq 1$ and $-1 \leq z\left(m_{-}\right)<0$.

Tables: Ratios of limiting to ordinary velocity squares as assigned by congruent parameter $z$

$$
\begin{aligned}
& \left(\begin{array}{cccccc}
z\left(m_{+}\right): & 1 & 0.6 & 0.2 & 10^{-2} & 10^{-4} \\
c_{1}^{2}\left(m_{+}\right) / v^{2}: & 1.5 & 3.2 & 12.46 & 259.3 & 25,980 \\
c_{2}^{2}\left(m_{+}\right) / v^{2}: & -3 & -4.76 & -13.46 & -269.3 & -25,981 \\
c_{3}^{2}\left(m_{+}\right) / v^{2}: & 1.5 & 1.07 & 1.006 & 1 & 1
\end{array}\right) \\
& \left(\begin{array}{cccccc}
z\left(m_{-}\right): & -1 & -0.6 & -0.2 & -10^{-2} & -10^{-4} \\
c_{1}^{2}\left(m_{-}\right) / v^{2}: & -3 & -4.76 & -13.46 & -260.3 & -25,981 \\
c_{2}^{2}\left(m_{-}\right) / v^{2}: & 1.5 & 3.7 & 12.46 & 269.3 & 25,980 \\
c_{3}^{2}\left(m_{-}\right) / v^{2}: & 1.5 & 1.07 & 1.006 & 1 & 1
\end{array}\right)
\end{aligned}
$$

From the Tables, one sees that the roles of particle limiting velocities $c_{1}$ and $c_{2}$ interchange when changing from $m_{+}=$ $m>0$ to $m_{-}=-m<0$ particle mass. Specifically, the square of the primary limiting velocity $c_{1}^{2}\left(m_{+}\right)>0$ got changed into the square of the obscure limiting velocity $c_{1}^{2}\left(m_{-}\right)<0$ and vice versa; the square of the obscure limiting velocity $c_{2}^{2}\left(m_{+}\right)<0$ got changed into the square of the primary limiting velocity $c_{2}^{2}\left(m_{-}\right)>0$.Also from the Tables one sees that changing $m_{+}=m>0$ to $m_{-}=-m<0, c_{3}^{2}$ remains numerically unchanged: $c_{3}^{2}\left(m_{+}\right) \rightarrow c_{3}^{2}\left(m_{-}\right)=c_{3}^{2}\left(m_{+}\right)$, the normal particle remains the normal particle. The implication of this fact is that the equality $c_{3}^{2}\left(m_{+}\right)=c_{3}^{2}\left(m_{-}\right)$makes the particle mass equally probable to be $m_{+}=m>0$ as well as $m_{-}=-m<0$ as we shall see shortly on the case of the muon neutrino.

Accepting that those flavor neutrinos $v(\alpha)$, with flavors $\alpha=e, \mu, \tau$, which, while in processes do not change the flavor, belong to normal particle classification with $c_{3}$ limiting velocities in the bicubic equation particle limiting velocity formalism, example of which is $v(\mu)$ from the OPERA velocity experiment (Adam et al., 2013). With this in mind an $\alpha$ flavor neutrino could in principle have either a positive or negative mass as just determined from discussing the Tables together with equality of (3.3) with (4.3). With these, for positive and negative neutrino masses one writes 


$$
\begin{aligned}
m_{+v}(\alpha) & >0, m_{-v}(\alpha)<0, m_{-v}(\alpha)=-m_{+v}(\alpha) ; \\
c_{3}^{2}\left(m_{+v}(\alpha)\right) & =c_{3}^{2}\left(m_{-v}(\alpha)\right)
\end{aligned}
$$

Relation (11) implies that a neutrino with either positive or negative mass has the same squared limiting velocity.

Here, the hypothesis that the flavor neutrino $v(\alpha)$ may be described either by positive $m_{+v}(\alpha)$ or negative $m_{-v}(\alpha)$ neutrino mass is tested on the case of the OPERA muon neutrino , $v(\mu)$, velocity experiment (Adam et al., 2013). For our purposes, we continue with data from Adam et al. (2013) as prepared and appearing in 'Soln (2014, 2016), with addition of a negative neutrino mass. In order to find $m_{-v}(\mu)$ one needs to have $m_{+v}(\mu)$. To arrive at this mass, as in 'Soln (2014, 2016), one starts with Gupta, Joshipura, and Patel, (2013) who with the freedom of spontaneous symmetry breaking arrives at the mass-state neutrino masses which are then used to deduce what the spontaneous symmetry should generate. Hence, following Gupta, Joshipura, and Patel, (2013), Harrison, Perkins, and Scott, (2002) use the neutrino tri-bimaxial neutrino matrix for flavor index (compare with 'Soln (2014)) yielding, among other flavor neutrino masses, $m_{+v}(\mu) c^{2}=0.76$ $e V$.Although, this result might be a little bit dated, it should serve as a useful model for showing the equivalence of negative and positive neutrino masses as far as the neutrino limiting velocity is concerned. Specifically, with the inclusion of this negative mass value and the energy from Khamehchi et al. (2017), we can write data relevant for OPERA muon neutrino velocity experiment in our notation,

$$
\begin{aligned}
E_{v}(\mu) & =17 \mathrm{GeV}, v_{v}(\mu) \simeq c, \\
m_{+v}(\mu) c^{2} & =+0.76 \mathrm{eV}, m_{-v}(\mu) c^{2}=-0.76 \mathrm{eV}
\end{aligned}
$$

where also - sign is present with the neutrino mass in order to demonstrates the equivalence of $m_{-v}(\mu) c^{2}$ with $m_{+v}(\mu) c^{2}$ in the evaluated single value $c_{3}^{2}\left(m_{+v}(\mu)\right)=c_{3}^{2}\left(m_{-v}(\mu)\right)$. Assuming that the velocity of the muon neutrino $v_{v}(\mu)$ satisfy

$$
\left(\frac{m_{ \pm v}(\mu) v_{v}(\mu)^{2}}{E_{v}(\mu)}\right)^{2} \ll 1
$$

which can be verified a posteriori, one solves simultaneously equations (3.3) and (4.3) approximately as in 'Soln (2014, 2016).

$$
c_{3}^{2}\left(m_{ \pm v}(\mu)\right)=v_{v}^{2}(\mu)\left[1+\left(\frac{m_{ \pm v}(\mu) c^{2}}{E_{v}(\mu)}\right)^{2}\left(\frac{v_{v}(\mu)}{c}\right)^{4}+O\left(\left(\frac{m_{ \pm v}(\mu) c^{2}}{E_{v}(\mu)}\right)^{4}\left(\frac{v_{v}(\mu)}{c}\right)^{8}\right)\right]
$$

Now with $\left(m_{ \pm v}(\mu) c^{2} / E_{v}(\mu)\right)^{2} \simeq 20.25 \times 10^{-24}$,one deduces,

$$
c_{3}^{2}\left(m_{+v}(\mu)\right)=c_{3}^{2}\left(m_{-v}(\mu)\right) \simeq v_{v}^{2}(\mu) \simeq c^{2}
$$

where, with very small error, $v_{v}(\mu)$ was measured to be the velocity of light $c$ in Adam et al. (2013).

What one would like to know is under which circumstances mass of the muon neutrino is positive or negative; that is to say, can one find criteria when neutrino has either $m_{+v}(\mu)$ or $m_{-v}(\mu)$ mass. More ambitious question to ask is: Do these masses alternate by changing into each other spontaneously? Further more, although limiting velocity square $c_{3}^{2}$ in (11.3) was calculated perturbatively, the equality of expression $c_{3}^{2}\left(m_{+v}(\mu)\right)=c_{3}^{2}\left(m_{-v}(\mu)\right)$ is exact due to equalities of $(3.3)$ and (4.3).

\section{Discussion and conclusion}

It is interesting how interrelated are particle positive and negative masses through their limiting velocities. Their jnterrelationships are uniquely influenced by the congruent parameter $z$ whose values are split into two branches defined by positive and negative mass values; between 0 and 1 , for positive masses and between -1 and 0 , for negative masses, respectively. The expressions for particle energies and momenta show formal similarities between positive and negative particle masses; their numerical values, however, show noticeable differences between the positive and negative particle masses.

Theoretically, and particularly from the Tables :'Ratios of limiting to ordinary velocity squares as assigned by congruent parameter $z$, one sees that when exchanging similarly numbered positive and negative particle masses, one exchanges primary to obscure, obscure to primary and normal to normal limiting particle velocities. The last case is relevant to the 
OPERA muon neutrino velocity experiment (Adam et al., 2013) as previously described in 'Soln, 2014, 2016), in which now the muon neutrino with either positive or negative mass yields the same normal particle limiting velocity $c$, consistent with measured result (Adam et al., 2013).

Of interest here is also as to how negative neutrino mass might fit into neutrino oscillations. This could possibly best be done with phenomenological approach to neutrino oscillations ('Soln, 2009) particularly with the short baseline ones ('Soln, 2011).

\section{References}

Śoln, J. (2009). Massive neutrinos, Lorentz invariance dominated Standaed Model and the phenomenological approach to neutrino oscillations. Physica Scripta, 80, 025101. arXiV: 0908. 1763 (2009).

Śoln, J. (2011). Cross-sections of long and short baseline neutrino and antineutrino oscillations of which some change the flavor. arXiv preprint arXiv:1106.0493.

Śoln, J. (2014). Theoretical particle limiting velocity from the bicubic equation: Neutrino example. Physics Essays, 27(3), 448. arXiV: 1403. 2683.

Śoln, J. (2015). Particle limiting velocities from the bicubic equation derived from the Einstein's kinematics: PeV electron case. Applied Physics Research, 7(4), 37.

Śoln, J. (2016). Limiting velocities of primary, obscure and normal particles: Self-annihilating obscure particle as an example of dark matter particle. Applied Physics Research, 8(5), 1.

Śln, J. (2017). Connecting dark matter particles with the primary, obscure and normal particles through implicit causality. Applied Physics Research, 9(3), 1.

Śoln, J. (2018). Positive and negative particle masses in the bicubic equation limiting particle velocity formalism. Applied Physics Research, 10(1), 14

Adam, T., Agafonova, N., Aleksandrov, A., Anokhina, A., Aoki, S., Ariga, A., ... \& Beretta, M. (2013). Measurement of the neutrino velocity with the OPERA detector in the CNGS beam using the 2012 dedicated data. Journal of High Energy Physics, 2013(1), 153.

Gaal, R. (2017). To disperse or not to disperse: Debating negative mass. APS News, 26(7), 1.

Gupta, S., Joshipura, A. S., \& Patel, K. M. (2013). How good is - symmetry after results on non-zero $\theta_{13}$ ? Journal of High Energy Physics, 2013(9), 35.

Harrison, P. F., Perkins, D. H., \& Scott, W. G. (2002). Tri-bimaximal mixing and the neutrino oscillation data. Physics Letters $B$, 530(1-4), 167-173.

Khamehchi, M. A., Hossain, K., Mossman, M. E., Zhang, Y., Busch, T., Forbes, M. M., \& Engels, P. (2017). Negativemass hydrodynamics in a spin-orbitCcoupled bose-einstein condensate. Physical review letters, $118(15), 155301$. arXiV: 1612.04055 .

\section{Copyrights}

Copyright for this article is retained by the author(s), with first publication rights granted to the journal.

This is an open-access article distributed under the terms and conditions of the Creative Commons Attribution license (http://creativecommons.org/licenses/by/4.0/). 